\title{
Bilinear Hilbert transform on measure spaces.
}

\author{
O. Blasco, ${ }^{*}$ M. Carro ${ }^{\dagger}{ }^{\top}$.A. Gillespie
}

\begin{abstract}
In this paper we obtain the boundedness of the periodic, discrete and Ergodic bilinear Hilbert transform, from $L^{p_{1}} \times L^{p_{2}}$ into $L^{p_{3}}$, where $1 / p_{1}+1 / p_{2}=1 / p_{3}$ and $p_{i} \geq 1$. The main techniques are a bilinear version of the transference method of Coifman and Weiss and certain discretization of bilinear operators. In the periodic case, we also obtain the boundedness for $2 / 3<p_{3}<1$.
\end{abstract}

\section{Introduction.}

If $T: \mathcal{S}(\mathbb{R}) \times \mathcal{S}(\mathbb{R}) \rightarrow \mathcal{S}^{\prime}(\mathbb{R})$ is a continuous bilinear operator which commutes with simultaneous translations then, in the distributional sense, $T$ can be represented as

$$
T(f, g)(x)=\int_{\mathbb{R}^{2}} \hat{f}(\xi) \hat{g}(\nu) m(\xi, \nu) e^{2 \pi i x(\xi+\nu)} d \xi d \nu,
$$

for Schwarzt test functions $f$ and $g$ belonging to $\mathcal{S}(\mathbb{R})$. It has been of great interest in the last decades to find conditions on the symbol $m$ such that $T$ extends to a bounded operator from $L^{p}(\mathbb{R}) \times L^{q}(\mathbb{R}) \rightarrow L^{r}(\mathbb{R})$ whenever $1 / r=1 / p+1 / q$ (see for example the works of [7], [11],[12], [15] or [18]). In particular, if

$$
T(f, g)(x)=\int_{\mathbb{R}^{n}} K(y) f(x-y) g(x+y) d y,
$$

where $K(y)=\frac{\Omega\left(y^{\prime}\right)}{|y|^{n}}, y^{\prime} \in \Sigma_{n-1}$ and $\Omega$ is an odd-function then

$$
T(f, g)(x)=\frac{1}{2} \int_{\Sigma_{n-1}} \Omega(\theta)\left(\int_{-\infty}^{\infty} f(x-t \theta) g(x+t \theta) \frac{d t}{t}\right) d \theta .
$$

*Partially supported by CICYT BFM2002-0416

†Partially supported by MTM2004-02299 and CURE 2001SGR 00069 
The operator inside the brackets $H_{\theta}(f, g)(x)=\int_{-\infty}^{\infty} f(x-t \theta) g(x+t \theta) \frac{d t}{t}$ is the so-called uni-directional bilinear Hilbert transform whose boundedness can be proved directly from the boundedness of the bilinear Hilbert transform

$$
H(f, g)(s)=\int_{-\infty}^{\infty} f(s-t) g(s+t) \frac{d t}{t}, s \in \mathbb{R} .
$$

This operator appeared for the first time in 1960, when A.P. Calderón was analyzing Cauchy integrals on Lipschitz curves and, in particular, the boundedness on $L^{2}(\mathbb{R})$ of the first commutator with a kernel $\frac{A(x)-A(y)}{(x-y)^{2}}$ where $A^{\prime} \in$ $L^{\infty}(\mathbb{R})$, and he needed to prove that the operator $H$ maps boundedly $L^{2}(\mathbb{R}) \times$ $L^{2}(\mathbb{R}) \rightarrow L^{1}(\mathbb{R})($ see $[6,17])$.

After several papers concerning the problem, M. Lacey and C. Thiele (see $[19,21,22])$ proved Calderón's conjecture showing that $H: L^{p}(\mathbb{R}) \times L^{q}(\mathbb{R}) \rightarrow$ $L^{r}(\mathbb{R})$ whenever $1 / p+1 / q=1 / r<3 / 2$. (see also $[13,14]$ ).

Since then, multilinear operators have become a matter of great interest in Harmonic Analysis.

In 2001, D. Fan and S. Sato ([10]) were able to show the boundedness of the bilinear Hilbert transform on the torus

$$
H_{\mathbb{T}}(f, g)(x)=\int_{\mathbb{T}} f(x-y) g(x+y) \cot (\pi y) d y,
$$

by transferring the result from $\mathbb{R}$. Their proof relies upon some DeLeeuw type transference methods for multilinear multipliers (see [9]). Similar techniques have been recently extended in [3] and [4].

Our aim in this paper will be to study the boundedness of the bilinear Hilbert transform in different measure spaces. In particular, we shall obtain the boundedness (on the same range but $p_{3} \geq 1$ ) of the discrete Hilbert transform

$$
H_{\mathbb{Z}}(a, b)(m)=\sum_{n \neq 0} \frac{a_{m-n} b_{n+m}}{n},
$$

of the ergodic Hilbert transform

$$
H_{T}(f, g)(x)=\sum_{n \neq 0} \frac{T^{n} f(x) T^{-n} g(x)}{n},
$$

where $T$ is an ergodic transformation acting on $L^{p_{i}}(\Omega)$ for a certain $\sigma$-finite measure space $\Omega$, and, hence also of

$$
H_{D}(f, g)(x)=\sum_{n \neq 0} \frac{f(x-n) g(x+n)}{n},
$$


and, we shall also give a new proof of the result of Fan and Sato about the boundedness of the bilinear Hilbert transform in the torus.

The main technique is based in the so-called transference method of R. Coifman and G. Weiss (see [8]). This method was introduced in 1977 and since then, it has been developed and extended by many other people (see [2] or [1]) and has shown to be an extremely useful tool to prove the boundedness of many operators defined on certain measure space assuming that we know the boundedness of a related convolution operator on certain group.

In 1996, L. Grafakos and G. Weiss (see [16]) proved a first result concerning a transference method for multilinear operators. They consider a multilinear operator $T$ defined on an amenable group $G$ by

$$
T\left(g_{1}, \ldots, g_{k}\right)(v)=\int_{G^{k}} K\left(u_{1}, \ldots, u_{k}\right) g_{1}\left(u_{1}^{-1} v\right) \ldots g_{k}\left(u_{k}^{-1} v\right) d \lambda\left(u_{1}\right) \ldots d \lambda\left(u_{k}\right),
$$

with $g_{j}$ in some dense subset of $L^{p_{j}}(G)$ and where $K$ is a kernel on $G^{k}$ which may not be integrable, and they are able to transfer the boundedness of $T: L^{p_{1}}(G) \times \ldots L^{p_{k}}(G) \rightarrow L^{p_{0}}(G)$ whenever $1 / p_{0}=1 / p_{1}+\ldots+1 / p_{k}$ to the boundedness of operator $\tilde{T}: L^{p_{1}}(\mu) \times \ldots L^{p_{k}}(\mu) \rightarrow L^{p_{0}}(\mu)$ where $(M, \mu)$ is a measure space and

$$
\tilde{T}\left(f_{1}, \ldots, f_{k}\right)(x)=\int_{G^{k}} K\left(u_{1}, \ldots, u_{k}\right)\left(R_{u_{1}}^{1} f_{1}\right)(x) \ldots\left(R_{u_{k}}^{k} f_{k}\right)(x) d \lambda\left(u_{1}\right) \ldots d \lambda\left(u_{k}\right),
$$

where $f_{j}$ is in some dense subset of $L^{p_{j}}(M)$, and $R^{j}: G \rightarrow \mathcal{B}\left(L^{p_{j}}(M)\right)$ $(j=0,1, \ldots, k)$ are representations which are connected through $R_{v}^{0} R_{u}^{j}=R_{u v}^{j}$ for all $u, v \in G$ and $1 \leq j \leq k$, and satisfy certain boundedness conditions.

In this paper, we shall develop a transference method for bilinear operators in the same spirit as the one started by Coifman and Weiss for linear operators, which will allow us to transfer the boundedness of bilinear operators such as the bilinear Hilbert transform on $\mathbb{R}$ to other groups, recovering the Fan and Sato transference result from our general principle. We shall restrict ourselves to the two variable case, to locally compact abelian groups $G$ and to integrable kernels (although our results will work in multilinear situation, amenable groups and more general kernels). A much more detailed study of this type of transference will be undertaken in [5]. Here, we shall be more concerned about the applications related to the bilinear Hilbert transform on measure spaces.

The second technique that we shall use concerns the discretization of bilinear operators. 


\section{Main techniques: Transference method and discretization}

\subsection{Transference method for bilinear operators}

Let $K \in L^{1}(G)$ be a kernel with compact support and let $1 \leq p_{1}, p_{2}<\infty$ and $0<p_{3}<\infty$ such that

$$
\frac{1}{p_{3}}=\frac{1}{p_{1}}+\frac{1}{p_{2}} \text {. }
$$

From now on, $p_{1}, p_{2}$ and $p_{3}$ will satisfy the above relation.

Consider the mapping

$$
B_{K}(\phi, \psi)(v)=\int_{G} \phi\left(u^{-1} v\right) \psi(u v) K(u) d m(u),
$$

for $\phi \in L^{p_{1}}(G)$ and $\psi \in L^{p_{2}}(G)$, where $m$ is the Haar measure on $G$, and let us define the transference operator $T_{K}: L^{p_{1}}(\mu) \times L^{p_{2}}(\mu) \rightarrow L^{p_{3}}(\mu)$ by

$$
T_{K}(f, g)(x)=\int_{G}\left(R_{u^{-1}}^{1} f\right)(x)\left(R_{u}^{2} g\right)(x) K(u) d m(u),
$$

where $R^{j}: G \rightarrow B\left(L^{p_{j}}(\mu)\right)$ are strongly continuous mappings for $j=1,2$. Then:

Theorem 2.1 Under the above conditions, if, for every $v \in G$, there exist $A_{j}>0$ such that

$$
\left\|R_{v}^{j} f\right\|_{L^{p_{j}}} \leq A_{j}\|f\|_{L^{p_{j}}}
$$

and there exists a strongly continuous mapping $R^{3}: G \rightarrow B\left(L^{p_{3}}(\mu)\right)$ satisfying that, for every $u, v \in G$ and every $f \in L^{p_{1}}(M)$ and $g \in L^{p_{2}}(M)$,

$$
R_{v}^{3}\left(R_{u^{-1}}^{1} f R_{u}^{2} g\right)=R_{v u^{-1}}^{1} f R_{v u}^{2} g,
$$

and such that, for every $v \in G$, there exists $B>0$ satisfying

$$
\|f\|_{L^{p_{3}(M)}} \leq B\left\|R_{v}^{3} f\right\|_{L^{p_{3}(M)}} .
$$

then, the bilinear operator $T_{K}: L^{p_{1}}(\mu) \times L^{p_{2}}(\mu) \rightarrow L^{p_{3}}(\mu)$ is bounded and it has norm bounded by $N_{p_{1}, p_{2}}(K) A_{1} A_{2} B$ where $N_{p_{1}, p_{2}}(K)$ stands for the norm of the bilinear map $B_{K}$ in the corresponding spaces. 
Proof. Using the continuity of $R_{v}^{3}$ and (2), we get that

$$
\begin{aligned}
R_{v}^{3}\left(T_{K}(f, g)\right) & =\int_{G} R_{v}^{3}\left(R_{u^{-1}}^{1} f R_{u}^{2} g\right) K(u) d m(u) \\
& =\int_{G} R_{v u^{-1}}^{1} f R_{v u}^{2} g K(u) d m(u),
\end{aligned}
$$

and by (3), we obtain that, for every $f \in L^{p_{1}}(\Omega), g \in L^{p_{2}}(\Omega)$, and every open set $V \subset G$,

$$
\left\|T_{K}(f, g)\right\|_{L^{p_{3}(\mu)}}^{p_{3}} \leq B^{p_{3}} \frac{1}{m(V)} \int_{V} \int_{\Omega}\left|R_{v}^{3} T_{K}(f, g)\right|^{p_{3}} d \mu d m(v) .
$$

Now, we can use similar arguments to those given in [8]. For any $\varepsilon>0$, let $V \in \mathcal{V}$ such that

$$
\max \left\{\frac{m(V C)}{m(V)}, \frac{m\left(V C^{-1}\right)}{m(V)}\right\} \leq 1+\varepsilon,
$$

with $C=\operatorname{supp} K$. Then,

$$
\begin{aligned}
& \left\|T_{K}(f, g)\right\|_{L^{p_{3}(\mu)}}^{p_{3}} \\
\leq & \frac{B^{p_{3}}}{m(V)} \int_{\Omega} \int_{V}\left|\int_{G}\left(R_{v u^{-1}}^{1} f\right) \chi_{V C^{-1}}\left(v u^{-1}\right)\left(R_{v u}^{2} g\right) \chi_{V C}(v u) K(u) d m(u)\right|^{p_{3}} d \mu d m(v) \\
\leq & B^{p_{3}} \frac{1}{m(V)} \int_{\Omega}\left\|B_{K}\left(R_{u}^{1} f \chi_{V C^{-1}}, R_{u}^{2} g \chi_{C V}\right)\right\|_{p_{3}}^{p_{3}} d \mu \\
\leq & B^{p_{3}} N_{p_{1}, p_{2}}(K)^{p_{3}} \\
\cdot & \left.\frac{1}{m(V)} \int_{\Omega}\left[\left(\int_{V C^{-1}}\left|R_{v}^{1} f\right|^{p_{1}} d m(v)\right)^{p_{3} / p_{1}}\left(\int_{V C}\left|R_{v}^{2} g\right|^{p_{2}} d m(v)\right)\right)^{p_{3} / p_{2}}\right] d \mu \\
\leq & B^{p_{3}} N_{p_{1}, p_{2}}(K)^{p_{3}} \frac{1}{m(V)}\left(\int_{V C^{-1}}\left\|R_{v}^{1} f\right\|_{L^{p_{1}}}^{p_{1}}\right)^{p_{3} / p_{1}}\left(\int_{V C}\left\|R_{v}^{2} g\right\|_{L^{p_{2}}}^{p_{2}}\right)^{p_{3} / p_{2}} \\
\leq & B^{p_{3}} A_{1}^{p_{3}} A_{2}^{p_{3}} N_{p_{1}, p_{2}}(K)^{p_{3}}(1+\varepsilon)\|f\|_{p_{1}}^{p_{3}}\|g\|_{p_{2}}^{p_{3}},
\end{aligned}
$$

from which the result follows.

\subsection{Discretization techniques}

Let us denote $A_{u}=u+A$ where $u \in \mathbb{R}$ and $A$ is an interval in $\mathbb{R}$ and let $I=(-1 / 4,1 / 4)$ and $p \geq 1$. Denote by $Q: L^{p}(\mathbb{R}) \rightarrow \ell^{p}(\mathbb{Z})$ the bounded 
operator defined by

$$
f \rightarrow\left(\int_{I_{n}} f(t) d t\right)_{n \in \mathbb{Z}}
$$

and by $P: \ell^{p}(\mathbb{Z}) \rightarrow L^{p}(\mathbb{R})$ the map defined by

$$
\left(a_{n}\right)_{n \in \mathbb{Z}} \rightarrow f=\sum_{n \in \mathbb{Z}} a_{n} \chi_{I_{n}} .
$$

Observe that $\|Q\|=2^{-1 / p^{\prime}}$ and $\|P\|=2^{-1 / p}$.

Proposition 2.2 Let $K$ be an integrable kernel in $L^{1}(\mathbb{R})$ and let us define

$$
K_{n}=\int_{I} \int_{\left(n+I_{u}\right) \cap\left(n-I_{u}\right)} K(t) d t d u .
$$

If

$$
T_{K}(f, g)(x)=\int_{\mathbb{R}} f(x-t) g(x+t) K(t) d t
$$

then

$$
Q T_{K}(P a, P b)(m)=T_{\left(K_{n}\right)}(a, b)(m)=\sum_{n \in \mathbb{Z}} a_{m-n} b_{m+n} K_{n} .
$$

In particular, for $p_{3} \geq 1$, one gets $\left\|T_{\left(K_{n}\right)}\right\|_{p_{1}, p_{2}} \leq \frac{1}{2}\left\|T_{K}\right\|_{p_{1}, p_{2}}$, where $\left\|T_{\left(K_{n}\right)}\right\|_{p_{1}, p_{2}}$ stands for the norm of the bilinear map $T_{\left(K_{n}\right)}$ from $\ell^{p_{1}}(\mathbb{Z}) \times \ell^{p_{2}}(\mathbb{Z})$ to $\ell^{p_{3}}(\mathbb{Z})$ and $\left\|T_{K}\right\|_{p_{1}, p_{2}}$ stands for the norm of the bilinear map $T_{K}$ from $L^{p_{1}}(\mathbb{R}) \times L^{p_{2}}(\mathbb{R})$ to $L^{p_{3}}(\mathbb{R})$.

\section{Proof:}

Given $a, b$ be finite sequences, we have that

$$
\begin{aligned}
T_{K}(P a, P b)(x) & =\sum_{n, m} a_{n} b_{m} T_{K}\left(\chi_{I_{n}}, \chi_{I_{m}}\right)(x) \\
& =\sum_{n, m} a_{n} b_{m} \int_{\left(x-I_{n}\right) \cap\left(-x+I_{m}\right)} K(t) d t \\
& =\sum_{n, m} a_{n} b_{m} \int_{(x-n+I) \cap(-x+m+I)} K(t) d t .
\end{aligned}
$$

Now, it is clear that $(x-n+I) \cap(-x+m+I) \neq \emptyset$ if and only if $\mid 2 x-(n+$ $m) \mid<1 / 2$, and hence, given $k \in \mathbb{Z}$ and $x \in I_{k},(x-n+I) \cap(-x+m+I) \neq \emptyset$ 
implies that $|2 k-(n+m)|<1$; that is $2 k=n+m$. Thus,

$$
\begin{aligned}
\int_{I_{k}} T_{K}(P a, P b)(x) d x & =\sum_{n, m} a_{n} b_{m} \int_{k+I} \int_{(x-n+I) \cap(-x+m-I)} K(t) d t d x \\
& =\sum_{n, m} a_{n} b_{m} \int_{I} \int_{\left(k-n+I_{u}\right) \cap\left(-k+m-I_{u}\right)} K(t) d t d u \\
& =\sum_{n+m=2 k} a_{n} b_{m} \int_{I} \int_{\left(k-n+I_{u}\right) \cap\left(k-n-I_{u}\right)} K(t) d t d u \\
& =\sum_{l \in \mathbb{Z}} a_{k-l} b_{k+l} \int_{I} \int_{\left(l+I_{u}\right) \cap\left(l-I_{u}\right)} K(t) d t d u,
\end{aligned}
$$

and therefore, for every $m \in \mathbb{Z}$,

$$
Q T_{K}(P a, P b)(m)=\sum_{n \in \mathbb{Z}} a_{m-n} b_{m+n} K_{n},
$$

as we wanted to see.

\section{Applications}

\subsection{Bilinear Hilbert transform on $\mathbb{T}$}

We shall apply our transference method to give a new proof of the result in [10] for the bilinear Hilbert transform on $\mathbb{T}$. For such a purpose take $G=\mathbb{R}$ with the Lebesgue measure, $(\Omega, \Sigma, \mu)$ the measure space $(\mathbb{T}, \mathcal{B}(\mathbb{T}), m)$ the Lebesgue measure on $\mathbb{T}$ and $R^{1}=R^{2}=R^{3}=R$, where

$$
\left(R_{u} f\right)\left(e^{i \theta}\right)=f\left(e^{i(\theta-u)}\right)
$$

Trivially $R^{j}, j=1,2,3$, satisfy conditions (1), (2) and (3).

Definition 3.1 A function $m \in L^{\infty}(\mathbb{R})$ is said to be normalized if $m_{n}=$ $\hat{\phi}_{n} * m$ is pointwise convergent to $m$ where $\phi_{n}(x)=\frac{1}{2 n} \chi_{[-n, n]} * \chi_{[-n, n]}$. 
Theorem 3.2 Let $K \in \mathcal{S}^{\prime}(\mathbb{R})$ such that $\hat{K}(\xi)=m(\xi)$ for some normalized function $m$. Let

$$
T_{K}(f, g)(x)=\int_{\mathbb{R}} \int_{\mathbb{R}} \hat{f}(\xi) \hat{g}(\nu) m(\xi-\nu) e^{i x(\xi+\nu)} d \xi d \nu,
$$

for $f, g \in \mathcal{S}$ and let

$$
\tilde{T}_{K}(P, Q)(x)=\sum_{k \in \mathbb{Z}} \sum_{k^{\prime} \in \mathbb{Z}} \hat{P}(k) \hat{Q}(k) m\left(k-k^{\prime}\right) e^{i x\left(k+k^{\prime}\right)},
$$

for $P$ and $Q$ trigonometric polynomials.

Then, if $T_{K}: L^{p_{1}}(\mathbb{R}) \times L^{p_{2}}(\mathbb{R}) \rightarrow L^{p_{3}}(\mathbb{R})$ is bounded, we have that

$$
\tilde{T}_{K}: L^{p_{1}}(\mathbb{T}) \times L^{p_{2}}(\mathbb{T}) \rightarrow L^{p_{3}}(\mathbb{T})
$$

is also bounded, if $p_{3} \geq 1$.

Proof. As in Lemma 3.5 of [8], let us take $\psi \in L^{2}(\mathbb{R})$ with compact support such that $\hat{\psi}(0)=1$ and let us define $K_{n}(x)=\left(m_{n} \hat{h}_{n}\right)(x)$ where $h_{n}(x)=$ $n \psi(n x)$. Then $K_{n} \in L^{1}(\mathbb{R})$, it has compact support and $\hat{K}_{n}(x) \rightarrow m(x)$ for all $x \in \mathbb{R}$.

Let

$$
T_{n}(f, g)(x)=\int_{\mathbb{R}} K_{n}(t) f(x-t) g(x+t) d t
$$

for $f, g \in \mathcal{S}(\mathbb{R})$.

We shall show first that $T_{n}: L^{p_{1}}(\mathbb{R}) \times L^{p_{2}}(\mathbb{R}) \rightarrow L^{p_{3}}(\mathbb{R})$ and $\sup _{n \in \mathbb{N}}\left\|T_{n}\right\|<$ $\infty$.

Now,

$$
\begin{aligned}
T_{n}(f, g)(x) & =\int_{\mathbb{R}} \hat{K}_{n}(\xi)[f(x-\cdot) g(x+\cdot)]^{\curlyvee}(\xi) d \xi \\
& =\int_{\mathbb{R}} \hat{h}_{n}(\xi) m_{n}(\xi)[f(x-\cdot) g(x+\cdot)] \curlyvee(\xi) d \xi \\
& =\int_{\mathbb{R}} h_{n}(t) A_{n}(t, x) d t
\end{aligned}
$$

where $A_{n}(x, t)=\left(\check{m}_{n} * f(x+\cdot) g(x-\cdot)\right)(t)$. We write

$$
\begin{aligned}
A_{n}(x, t) & =\int_{\mathbb{R}} \check{m}_{n}(y) f(x+t-y) g(x-t+y) d y \\
& =\int_{\mathbb{R}} m_{n}(y)[f(x+t-\cdot) g(x-t+\cdot)] \check{d} d y \\
& =\int_{\mathbb{R}}\left(\int_{\mathbb{R}} \hat{\phi}_{n}(z) m(y-z) d z\right)[f(x+t-\cdot) g(x-t+\cdot)] \check{d} d y \\
& =\int_{\mathbb{R}} \hat{\phi}_{n}(z)\left(\int_{\mathbb{R}} m(y-z)[f(x+t-\cdot) g(x-t+\cdot)] \check{c} d y\right) d z
\end{aligned}
$$


Now, observe that

$$
\begin{aligned}
B(z, x, t) & =\int_{\mathbb{R}} m(y-z)[f(x+t-\cdot) g(x-t+\cdot)] \backsim d y \\
& =\int_{\mathbb{R}} K(y) e^{2 \pi i z y} f(x+t-y) g(x-t+y) d y \\
& =\int_{\mathbb{R}} K(y) F_{z, t}(x-y) G_{z, t}(x+y) d y
\end{aligned}
$$

where $F_{z, t}(u)=e^{\pi i z u} f(u+t)$ and $G_{z, t}(u)=e^{\pi i z u} g(u-t)$.

Therefore,

$$
T_{n}(f, g)(x)=\int_{\mathbb{R}} h_{n}(t)\left(\int_{\mathbb{R}} \hat{\phi}_{n}(z) B_{K}\left(F_{z, t}, G_{z, t}\right)(x) d z\right) d t
$$

and, hence, since

$$
\left\|B_{K}\left(F_{z, t}, G_{z, t}\right)\right\|_{p_{3}} \leq C\left\|F_{z, t}\right\|_{p_{1}}\left\|G_{z, t}\right\|_{p_{2}} \leq C\|f\|_{p_{1}}\|g\|_{p_{2}},
$$

and $p_{3} \geq 1$, we obtain that

$$
\left\|T_{n}(f, g)\right\|_{p_{3}} \leq C\left\|h_{n}\right\|_{1}\left\|\hat{\phi}_{n}\right\|_{1}\|f\|_{p_{1}}\|g\|_{p_{2}} .
$$

Now, we can apply Theorem 2.1 with $R_{u} P(\theta)=P(\theta-u)$, to get that the transferred bilinear operator

$$
\tilde{T}_{n}(P, Q)(\theta)=\int_{\mathbb{T}} \tilde{K}_{n}(u) P(\theta-u) Q(\theta+u) d u,
$$

where $\tilde{K}_{n}(u)=\sum_{m \in \mathbb{Z}} K_{n}(m+u)$, is bounded from $L^{p_{1}}(\mathbb{T}) \times L^{p_{2}}(\mathbb{T}) \rightarrow L^{p_{3}}(\mathbb{T})$ and the norms are uniformly bounded for $n \in \mathbb{N}$.

To finish the proof observe that if $e_{k}(\theta)=e^{i k \theta}$ then

$$
\tilde{T}_{n}\left(e_{k}, e_{k^{\prime}}\right)=e_{k} e_{k^{\prime}} \int_{\mathbb{R}} K_{n}(u) e^{i u\left(k^{\prime}-k\right)} d u=e_{k+k^{\prime}} m_{n}\left(k-k^{\prime}\right) \hat{h}_{n}\left(k-k^{\prime}\right),
$$

and hence,

$$
\lim _{n \rightarrow \infty} T_{n}\left(e_{k}, e_{k^{\prime}}\right)=e_{k+k^{\prime}} m\left(k-k^{\prime}\right)=\tilde{T}_{K}\left(e_{k}, e_{k^{\prime}}\right) .
$$

Therefore, by linearity, density and Fatou's lemma, we obtain the result

Now, in order to avoid the condition of $p_{3} \geq 1$ in the case of the bilinear Hilbert transform, we need the following lemma that follows from the boundedness of the corresponding maximal bilinear Hilbert operator (see [20]). 
Lemma 3.3 Let $0<A<A^{\prime} \leq \infty$. Define $K_{A, A^{\prime}}(x)=\frac{1}{x} \chi_{A<|x|<A^{\prime}}(x)$. Let

$$
B_{A, A^{\prime}}(f, g)(x)=\int_{\mathbb{R}} f(x-t) g(x+t) K_{A, A^{\prime}}(t) d t
$$

and let $\left\|B_{A, A^{\prime}}\right\|_{p_{1}, p_{2}}$ denote the norm as bounded bilinear map from $L^{p_{1}}(\mathbb{R}) \times$ $L^{p_{2}}(\mathbb{R})$ into $L^{p_{3}}(\mathbb{R})$. Then

$$
\sup _{A, A^{\prime}}\left\|B_{A, A^{\prime}}\right\|_{p_{1}, p_{2}}<\infty .
$$

Let us give an easy proof of the above lemma in the case $p_{3} \geq 1$. To this end, we first need the following lemma.

Lemma 3.4 Let $p_{3} \geq 1$. Let $h_{1}, h_{2} \in L^{1}(\mathbb{R})$ and define $m(\xi)=\operatorname{sign}(\xi) \hat{h_{1}}(\xi)+$ $\hat{h}_{2}(\xi)$. If

$$
B_{h_{1}, h_{2}}(f, g)(x)=\int_{\mathbb{R}} \int_{\mathbb{R}} \hat{f}(\xi) \hat{g}(\eta) m(\xi-\eta) e^{i(\xi+\eta) x} d \xi d \eta
$$

Then $B_{h}$ is bounded from $L^{p_{1}}(\mathbb{R}) \times L^{p_{2}}(\mathbb{R})$ into $L^{p_{3}}(\mathbb{R})$ and $\left\|B_{h_{1}, h_{2}}\right\|_{p_{1}, p_{2}} \leq$ $\|H\|_{p_{1}, p_{2}}\left\|h_{1}\right\|_{1}+\left\|h_{2}\right\|_{1}$.

\section{Proof:}

$$
\begin{aligned}
B_{h_{1}, h_{2}}(f, g)(x) & =\int_{\mathbb{R}} \int_{\mathbb{R}} \hat{f}(\xi) \hat{g}(\eta) m(\xi-\eta) e^{i(\xi+\eta) x} d \xi d \eta \\
& =\int_{\mathbb{R}} \int_{\mathbb{R}} \hat{f}(\xi) \hat{g}(\eta) \operatorname{sign}(\xi-\eta)\left(\int_{\mathbb{R}} h_{1}(y) e^{-i(\xi-\eta) y} d y\right) e^{i(\xi+\eta) x} d \xi d \eta \\
& +\int_{\mathbb{R}} \int_{\mathbb{R}} \hat{f}(\xi) \hat{g}(\eta)\left(\int_{\mathbb{R}} h_{2}(y) e^{-i(\xi-\eta) y} d y\right) e^{i(\xi+\eta) x} d \xi d \eta \\
& =\int_{\mathbb{R}}\left(\int_{\mathbb{R}} \int_{\mathbb{R}} \hat{f}(\xi) e^{-i \xi y} \hat{g}(\eta) e^{i \eta y} \operatorname{sgn}(\xi-\eta) e^{i(\xi+\eta) x} d \xi d \eta\right) h_{1}(y) d y \\
& +\int_{\mathbb{R}}\left(\int_{\mathbb{R}} \int_{\mathbb{R}} \hat{f}(\xi) e^{-i \xi(y-x)} \hat{g}(\eta) e^{i \eta(y+x)} d \xi d \eta\right) h_{2}(y) d y \\
& =\int_{\mathbb{R}} H\left(f_{y}, g_{-y}\right)(x) h_{1}(y) d y+\int_{\mathbb{R}} f(x-y) g(x+y) h_{2}(y) d y
\end{aligned}
$$

where $f_{x}(y)=f(y-x)$. Now using the boundedness of the bilinear Hilbert transform, Hölder inequality and the integrability of $h_{1}$ and $h_{2}$ one gets the result.

Proof of Lemma 3.3 for $p_{3} \geq 1$ : It is known that

$$
m_{A, A^{\prime}}(\xi)=\hat{K}_{A, A^{\prime}}(\xi)=\operatorname{sign}(\xi) \int_{A}^{A^{\prime}} \frac{\sin (|\xi| u)}{u} d u
$$


and hence,

$$
m_{A, A^{\prime}}(\xi)=m(A \xi)-m\left(A^{\prime} \xi\right)
$$

where $m(\xi)=\operatorname{sign}(\xi) \int_{1}^{\infty} \frac{\sin (|\xi| u)}{u} d u$.

Denoting by $K(x)=K_{1, \infty}(x)=\frac{1}{x} \chi_{\{|x|>1\}}(x)$ and $Q(x)=\frac{x}{1+x^{2}}$, we have that $K-Q=h \in L^{1}(\mathbb{R})$. In particular,

$$
m(\xi)=\hat{K}(\xi)=-i \operatorname{sign}(\xi) \hat{P}(\xi)+\hat{h}(\xi),
$$

where $P(x)=\frac{1}{1+x^{2}}$ is the Poisson kernel.

Then,

$$
m_{A, A^{\prime}}(\xi)=-i \operatorname{sign}(\xi)\left(\hat{P}_{A}(\xi)-\hat{P}_{A^{\prime}}(\xi)\right)+\hat{h}_{A}(\xi)-\hat{h}_{A^{\prime}}(\xi),
$$

where, as usual, $f_{A}(x)=\frac{1}{A} f\left(\frac{x}{A}\right)$.

Finally, we can apply Lemma 3.4 to obtain that

$$
\left\|B_{A, A^{\prime}}\right\|_{p_{1}, p_{2}} \leq\|H\|_{p_{1}, p_{2}}\left\|P_{A}-P_{A^{\prime}}\right\|_{1}+\left\|h_{A}-h_{A^{\prime}}\right\|_{1} \leq 2\|H\|_{p_{1}, p_{2}}+2\|h\|_{1},
$$

as we wanted to see.

As a consequence of the previous result, we obtain the following ([10]).

Corollary 3.5 The bilinear Hilbert transform on the torus

$$
H_{\mathbb{T}}(f, g)(x)=\int_{\mathbb{T}} f(x-y) g(x+y) \cot (\pi y) d y,
$$

is bounded from $L^{p_{1}}(\mathbb{T}) \times L^{p_{2}}(\mathbb{T})$ into $L^{p_{3}}(\mathbb{T})$ whenever $1 / p_{1}+1 / p_{2}=1 / p_{3}<$ $3 / 2$.

Proof: Let us take $A=1 / N$ and $A^{\prime}=N$ in Lemma 3.3. Then, since the corresponding kernel $K_{A, A^{\prime}}$ is in $L^{1}$ with compact support, we can apply our transference argument and this lemma to obtain that the operator

$$
\tilde{T}_{K}^{N}(P, Q)(x)=\sum_{k \in \mathbb{Z}} \sum_{k^{\prime} \in \mathbb{Z}} \hat{P}(k) \hat{Q}(k) m_{1 / N, N}\left(k-k^{\prime}\right) e^{i x\left(k+k^{\prime}\right)},
$$

for $P$ and $Q$ trigonometric polynomials, satisfies that

$$
\tilde{T}_{K}^{N}: L^{p_{1}}(\mathbb{T}) \times L^{p_{2}}(\mathbb{T}) \rightarrow L^{p_{3}}(\mathbb{T})
$$

uniformly in $N$. Letting $N$ goes to infinity, we obtain the result. 


\subsection{Bilinear Hilbert transform on $\mathbb{Z}$}

Using now the discretization technique of section 2.2, we obtain the following result, whenever $p_{3} \geq 1$.

Proposition 3.6 Let $N \in \mathbb{N}$, and let us define the truncated discrete bilinear Hilbert transform by

$$
H_{\mathbb{Z}, N}(a, b)(m)=\sum_{k \neq 0,|n| \leq N} \frac{a_{m-n} b_{m+n}}{n} .
$$

Then,

$$
\sup _{N \in \mathbb{N}}\left\|H_{\mathbb{Z}, N}\right\|_{p_{1}, p_{2}}<\infty
$$

Proof: Let us apply Lemma 3.3 with $K=K_{\frac{1}{2}, N-\frac{1}{2}}$ and Proposition 2.2, to obtain that

$$
T_{N}(a, b)(m)=\sum_{n \in \mathbb{Z}} a_{m-n} b_{m+n} K_{n}
$$

is bounded from $\ell^{p_{1}}(\mathbb{Z}) \times \ell^{p_{2}}(\mathbb{Z})$ into $\ell^{p_{3}}(\mathbb{Z})$. Let us now compute $K_{n}$ in this particular case:

$$
\begin{aligned}
K_{n} & =\int_{I} \int_{\left(n+I_{u}\right) \cap\left(n-I_{u}\right)} K(t) d t d u \\
& =\int_{I} \int_{\left(n+I_{u}\right) \cap\left(n-I_{u}\right) \cap\left(\frac{1}{2}, N-\frac{1}{2}\right)} \frac{d t}{t} d u+\int_{I} \int_{\left(n+I_{u}\right) \cap\left(n-I_{u}\right) \cap\left(-N+\frac{1}{2},-\frac{1}{2}\right)} \frac{d t}{t} d u .
\end{aligned}
$$

Observe that for $u \in I$, we have that $\left(n+I_{u}\right) \cap\left(n-I_{u}\right) \subset\left(n-\frac{1}{2}, n+\frac{1}{2}\right)$, and hence $K_{n}=0$ if $|n| \geq N$ and $K_{0}=0$.

For $1 \leq n<N$, we can write

$$
K_{n}=2 \int_{0}^{1 / 4} \int_{\left(n+u-\frac{1}{4}, n+u+\frac{1}{4}\right) \cap\left(n-u-\frac{1}{4}, n-u+\frac{1}{4}\right) \cap\left(\frac{1}{2}, N-\frac{1}{2}\right)} \frac{d t}{t} d u,
$$

and if $0<u<\frac{1}{4}$, we obtain that

$$
\left(n+u-\frac{1}{4}, n+u+\frac{1}{4}\right) \cap\left(n-u-\frac{1}{4}, n-u+\frac{1}{4}\right)=\left(n+u-\frac{1}{4}, n-u+\frac{1}{4}\right) .
$$


Hence,

$$
\begin{aligned}
K_{n} & =2 \int_{0}^{1 / 4} \log \left(\frac{n-u+\frac{1}{4}}{n+u-\frac{1}{4}}\right) d u=2 \int_{0}^{1 / 4} \log \left(\frac{n+v}{n-v}\right) d v \\
& =2 n \int_{0}^{1 / 4 n} \log \left(\frac{1+x}{1-x}\right) d x .
\end{aligned}
$$

Integrating by parts, we obtain

$$
\begin{gathered}
\int_{0}^{1 / 4 n} \log \left(\frac{1+x}{1-x}\right) d x=\frac{1}{4 n} \log \left(\frac{1+\frac{1}{4 n}}{1-\frac{1}{4 n}}\right)-\int_{0}^{1 / 4 n} \frac{2 x}{1-x^{2}} d x \\
=\frac{1}{4 n} \log \left(\frac{1+\frac{1}{4 n}}{1-\frac{1}{4 n}}\right)+\log \left(1-\frac{1}{16 n^{2}}\right),
\end{gathered}
$$

and hence,

$$
K_{n}=\frac{1}{2} \log \left(\frac{n+\frac{1}{4}}{n-\frac{1}{4}}\right)-2 n \log \left(\frac{16 n^{2}}{16 n^{2}-1}\right) .
$$

Since $\log (1+x)=x+O\left(x^{2}\right),(x \rightarrow 0)$, we finally get

$$
\begin{aligned}
K_{n} & =\frac{1}{4 n-1}+O\left(\frac{1}{\left(n-\frac{1}{4}\right)^{2}}\right)+\frac{2 n}{16 n^{2}-1}+2 n O\left(\frac{1}{\left(16 n^{2}-1\right)^{2}}\right) \\
& =\frac{6 n+1}{16 n^{2}-1}+O\left(\frac{1}{n^{2}}\right)=\frac{3}{8 n}+O\left(\frac{1}{n^{2}}\right) .
\end{aligned}
$$

The case $-N \leq n \leq-1$ is obtained similarly, and the result follows from (5).

\subsection{Ergodic Bilinear Hilbert transform}

The idea now is to transfer the boundedness of the discrete bilinear Hilbert transform to a measure space using our transference result.

Let $G=\mathbb{Z}$ and let $(\Omega, \Sigma, \mu)$ be a $\sigma$-finite measure space. Let $T$ be a bounded and invertible operator acting on $L^{p_{i}}(\Omega)$, such that

$$
\max \left(\left\|T^{-1}\right\|_{L\left(L^{\left.p_{i}(\Omega)\right)}\right.},\|T\|_{L\left(L^{\left.p_{i}(\Omega)\right)}\right)} \leq 1,\right.
$$


for $i=1,2$. Let us assume that there exists a bounded and invertible operator $S$ acting on $L^{p_{3}}(\Omega)$, such that $\max \left(\left\|S^{-1}\right\|_{L\left(L^{p_{3}}(\Omega)\right)},\|S\|_{L\left(L^{p_{3}}(\Omega)\right)}\right) \leq 1$ and such that

$$
S^{m}\left(T^{n} f T^{-n} g\right)=T^{m+n} f T^{m-n} g .
$$

Then:

Theorem 3.7 The bilinear ergodic Hilbert transform

$$
H_{T}(f, g)(x)=\sum_{n \neq 0} \frac{T^{n} f(x) T^{-n} g(x)}{n}
$$

is bounded from $L^{p_{1}}(\Omega) \times L^{p_{2}}(\Omega)$ into $L^{p_{3}}(\Omega)$ whenever $1 / p_{1}+1 / p_{2}=1 / p_{3} \leq$ 1 .

Proof. It is trivial to see that if we take $R_{n}^{1}=R_{n}^{2}=T^{n}$ and $R_{n}^{3}=S^{n}$ and use (6) then conditions (1), (2) and (3) hold and hence, we can transfer, using Theorem 2.1, the boundedness of the truncated discrete bilinear Hilbert transform proved in Proposition 3.6, to show that, in fact,

$$
H_{T}^{N}(f, g)(x)=\sum_{n \neq 0, n=-N}^{N} \frac{T^{n} f(x) T^{-n} g(x)}{n}
$$

is bounded uniformly in $N$. From this, the result follows.

In particular, using $T f(x)=f(x-1)$ and $S=T$ one obtains the following:

Corollary 3.8 The bilinear Hilbert transform

$$
H(f, g)(x)=\sum_{n \neq 0} \frac{f(x-n) g(x+n)}{n}
$$

is bounded from $L^{p_{1}}(\mathbb{R}) \times L^{p_{2}}(\mathbb{R})$ into $L^{p_{3}}(\mathbb{R})$ whenever $1 / p_{1}+1 / p_{2}=1 / p_{3} \leq$ 1.

\section{References}

[1] Berkson,E., Paluszynski, M., Weiss, G., Transference couples and their applications to convolution operators and maximal operators. Lecture Notes in Pure and Applied Math. 175 [1996], pp. 69-84. 
[2] Berkson, E., Gillespie, T.A., Transference and extension of Fourier Multipliers for $L^{p}(\mathbb{T})$. J. London Math. Soc. 41 [1990], pp. 472-488.

[3] Blasco, O. Bilinear multipliers and transference. Preprint.

[4] Blasco, O., Villarroya, F., Transference of bilinear multipliers on Lorentz spaces. Illinois J. Math. 47 (4) [2004], pp. 1327-1343.

[5] Blasco, O., Carro, M., Gillespie, T.A., A general principle for bilinear operators. In preparation.

[6] Calderón A.P., Commutators of singular integral operators. Proc. Natl. Acad. Sci. USA, 53 [1977], pp. 1092-1099.

[7] Coifman R.R., Meyer Y. Au delà des operatateurs pseudo-differentiels, Asterisque 57 [1978]

[8] Coifman R.R., Weiss W. Transference Methods in Analysis. Regional Conference Series in Mathematics 31, A.M.S. (1977)

[9] DeLeeuw, K., On $L^{p}$ Multipliers, Ann. of Math. 81 [1969] pp. 364-379.

[10] Fan, D., Sato, S., Transference of certain multilinear multiplier operators. J. Austral. Math. Soc 70 [2001] pp. 37-55.

[11] Gilbert J., Nahmod A., Bilinear operators with non-smooth symbols. J. Fourier Anal. Appl. 7 [2001], pp. 435-467.

[12] Gilbert J., Nahmod A., Boundedness of bilinear operators with nonsmooth symbols Math. Res. Lett. 7 [2000], pp. 767-778

[13] Grafakos L., Li X., Uniform bounds for the bilinear Hilbert transform I To appear in Ann. of Math.

[14] Grafakos L., Li X., Uniform bounds for the bilinear Hilbert transform II Preprint.

[15] Grafakos L.,Torres, R. H., Multilinear Calderón-Zygmund theory. Adv. Math. 165 [2002], pp. 124-164.

[16] Grafakos, L., and Weiss, G., Transference of multilinear operators. Illinois J. Math. 40 [1996], pp. 344-351. 
[17] Jones P., Bilinear singular integrals and maximal functions, Havin and Nikolski (Eds.), Linear and Complex Analysis Problem Book 3, Part 1. Springer LNM 1573, [1994].

[18] Kenig, C. E., Stein, E.M., Multilinear estimates and fractional integration. Math. Res. Lett. 6 [1999], pp. 1-15.

[19] Lacey M., Thiele C., $L^{p}$ bounds on the bilinear Hilbert transform for $2<p<\infty$. Ann. Math. 146 [1997], pp. 693-724.

[20] Lacey M., Thiele C., The bilinear maximal function map into $L^{p}$ for $2 / 3<p \leq 1$. Ann. Math. 151 [2000], pp. 35-57 .

[21] Lacey, M. and Thiele, C. Weak bounds for the bilinear Hilbert transform on $L^{p}$. Documenta Mathematica, extra volume ICM 1-1000, [1997].

[22] Lacey, M., Thiele, C., On Calderón's conjecture. Ann. Math. 1492 [1999], pp. 475-496

Departamento de Análisis Matemático

Universitad de Valencia, Burjassot, 46100-Valencia, (SPAIN)

E-mail: oscar.blasco@uv.es

Departament de Matemàtica Aplicada i Anàlisi

Universitat de Barcelona, E-08071 Barcelona, (SPAIN)

E-mail: carro@ub.edu

Department of Mathematics and Statistics

University of Edinburgh, Edinburgh EH9 3JZ, (SCOTLAND)

E-mail: alastair@maths.ed.ac.uk 